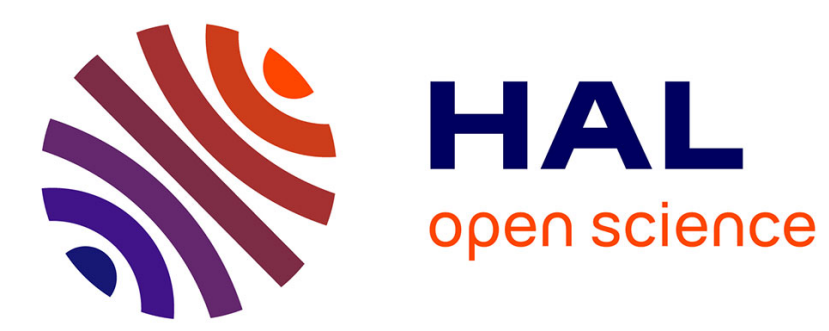

\title{
BAYESIAN FORMULATIONS FOR FORCE RECONSTRUCTION PROBLEMS
}

\author{
Mathieu Aucejo, Olivier de Smet
}

\section{To cite this version:}

Mathieu Aucejo, Olivier de Smet. BAYESIAN FORMULATIONS FOR FORCE RECONSTRUCTION PROBLEMS. 2nd International Conference on Uncertainty Quantification in Computational Sciences and Engineering, UNCECOMP 2017, Jun 2017, Rhodes Island, Greece. hal-02068535

\section{HAL Id: hal-02068535 https://hal.science/hal-02068535}

Submitted on 15 Mar 2019

HAL is a multi-disciplinary open access archive for the deposit and dissemination of scientific research documents, whether they are published or not. The documents may come from teaching and research institutions in France or abroad, or from public or private research centers.
L'archive ouverte pluridisciplinaire HAL, est destinée au dépôt et à la diffusion de documents scientifiques de niveau recherche, publiés ou non, émanant des établissements d'enseignement et de recherche français ou étrangers, des laboratoires publics ou privés. 


\title{
BAYESIAN FORMULATIONS FOR FORCE RECONSTRUCTION PROBLEMS
}

\author{
Mathieu Aucejo $^{1}$ and Olivier De Smet ${ }^{1}$ \\ ${ }^{1}$ Structural Mechanics and Coupled Systems Laboratory - Conservatoire National des Arts et Mtiers \\ 2 rue Conté, 75003 Paris \\ e-mail: \{mathieu.aucejo, olivier.desmet\}@lecnam.net
}

Keywords: Force reconstruction, Bayesian inference, Generalized Gaussian priors, MCMC.

\begin{abstract}
To identify mechanical sources acting on a structure, Tikhonov-like regularizations are generally used. These approaches, however, only provide point estimates, meaning that the uncertainty about the regularized solution is not quantified. In practice, such information is essential to guarantee the quality of reconstructed sources. In this contribution, three possible Bayesian formulations of the source identification problem are presented and their limitations discussed. To assess the posterior uncertainty on the parameters appearing in each formulation given a simulated vibration field and a mechanical model, a Gibbs sampler is implemented. The proposed numerical validations highlight the practical interest of these formulations in terms of parameters estimations and posterior uncertainty quantification.
\end{abstract}




\section{INTRODUCTION}

In structural dynamics, most of the research papers are generally focused on the modeling of the dynamic response of structures subject to perfectly determined excitation sources. However, the latter are only roughly or partially known in practice. As a result, an error in the definition of the excitation vector is propagated to the dynamic response through the model and can have a significant impact on the subsequent mechanical analysis. However, direct measurement of excitation sources can be practically unfeasible. A possible alternative is to perform indirect measurements using a model of the dynamic behavior of the studied structure and accessible quantities such as displacement or acceleration fields. Unfortunately, the reconstruction of mechanical sources from vibration measurements is an ill-posed inverse problem. A classical approach to bypass this difficulty consists in constraining the space of solutions by using prior information on the noise and the sources to reconstruct. A convenient and efficient way to deal with such prior information is the Bayesian framework, because it allows combining both probabilistic and mechanical data. The most widespread and popular approaches deriving from Bayesian statistics are certainly Tikhonov-like regularizations [1, 2, 3]. Although widely used and deeply studied, these methods generally provide only point estimates. In other words, there is no information on the uncertainty about the regularized solution given the measured data and the mechanical model. However, such information is of primary interest for industrial applications, in which it is essential to guarantee the quality of obtained results. In the present contribution, three possible Bayesian formulations of the source identification problem, based on the use of Generalized Gaussian distributions, Gamma and truncated Gamma distributions, are proposed. The limitations of each formulation are discussed from the identification of two close point forces acting on a free-free beam. To assess the posterior uncertainty on the parameters appearing in each formulation given a simulated vibration field and a mechanical model, a Gibbs sampler [4], including Hamiltonian Monte Carlo [5] updates, is implemented to perform the inference. The proposed MCMC procedure is detailed in this contribution. The proposed numerical validations highlight the practical interest of the proposed formulations in terms of parameters estimations and posterior uncertainty quantification.

\section{BAYESIAN FORMULATIONS OF THE RECONSTRUCTION PROBLEM}

This section aims at introducing the three formulations of the Bayesian force reconstruction problem as well as the related parameters. To render this section more didactic, each formulation is applied on an academic test case in order to better highlight its advantages and limitations. The MCMC algorithm used to perform the inferences will be detailed in section 3 .

\subsection{Description of the benchmark test case}

Before presenting the three Bayesian formulations of the reconstruction problem, we propose to introduce the test case that will serve as a benchmark to analyze and compare each of them. The studied structure is a free-free steel beam with dimensions $1 \times 0.03 \times 0.01 \mathrm{~m}^{3}$ excited by two point forces of unit amplitude at $350 \mathrm{~Hz}$. The coordinate of the point forces, measured from the left end of the beam, are $x_{1}=0.6 \mathrm{~m}$ and $x_{2}=0.7 \mathrm{~m}$ [see Fig. 1]].

To perform the reconstruction, a model has to be derived to relate the measured vibration field and the excitation field. For this purpose, let us consider the practical situation where the vibration field $\mathbf{X}$, measured over the surface of a structure, is caused by an unknown excitation field $\mathbf{F}$. If the structure is linear and time invariant, its dynamic behavior is completely deter- 


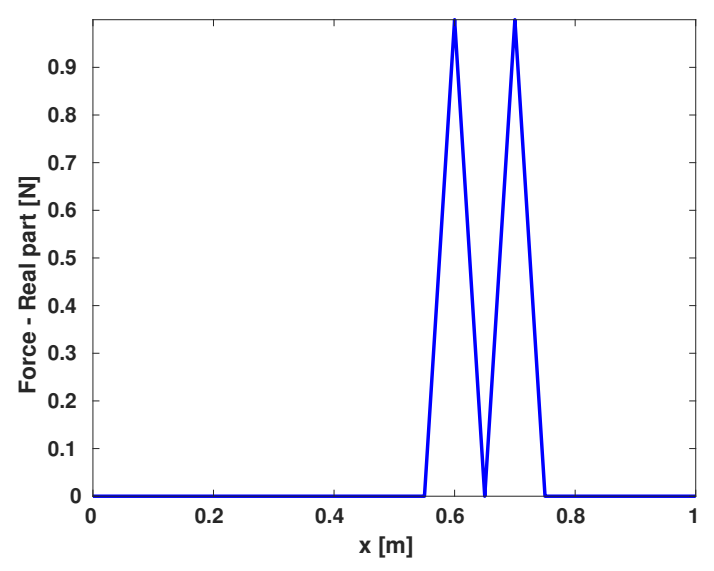

Figure 1: Reference force field

mined by the transfer functions matrix $\mathbf{H}$, relating the vibration field $\mathbf{X}$ to the excitation field $\mathbf{F}$, so that:

$$
\mathbf{X}=\mathbf{H F}+\mathbf{N}
$$

where $\mathbf{N}$ is the measurement noise vector.

A finite element model of the beam made up with 20 plane beam elements has been used to compute the transfer functions matrix $\mathbf{H}$ by assuming that only bending motions are measurable. In other words, the transfer functions matrix is dynamically condensed over the measurable dofs, corresponding here to the bending motions. To simulate the measured vibration field $\mathbf{X}$, Eq. (1) is applied using a noise vector $\mathbf{N}$ corresponding to a Gaussian white noise, which is calculated assuming a signal-to-noise ratio equal to $34 \mathrm{~dB}$. Finally, it is worth mentioning that the model defined in Eq. (1) is the basis of the proposed Bayesian formulations, meaning that the transfer functions matrix $\mathbf{H}$ is also used in the inverse problem. In other words, modeling errors are not considered here.

\subsection{Standard Bayesian formulation}

Formally, the Bayesian paradigm considers all the parameters of the problem as random variables. Consequently, the uncertainty on each parameter is modeled by a probability distribution, describing the state of knowledge or the prior on this parameter. From a mathematical standpoint, the Bayesian reconstruction problem relies on the Bayes' rule:

$$
p(\mathbf{F} \mid \mathbf{X}) \propto p(\mathbf{X} \mid \mathbf{F}) p(\mathbf{F}),
$$

where:

- $p(\mathbf{F} \mid \mathbf{X})$ is the posterior probability distribution, representing the probability of observing $\mathbf{F}$ given a vibration field $\mathbf{X}$. It defines what it is known about the excitation field $\mathbf{F}$ after making vibration measurements;

- $p(\mathbf{X} \mid \mathbf{F})$ is the likelihood function, representing the probability of measuring $\mathbf{X}$ given an excitation field $\mathbf{F}$. It reflects the uncertainty related to the measurement of the vibration field $\mathbf{X}$; 
- $p(\mathbf{F})$ is the prior probability distribution, representing our knowledge on the unknown excitation field $\mathbf{F}$ before measuring the vibration field $\mathbf{X}$.

Generally, the quality of the force reconstruction strongly depends on the choice of the likelihood function and the prior probability distribution. That is why, the choices made in this contribution have to be carefully explained.

\subsubsection{Choice of the likelihood function}

The likelihood function reflects the uncertainty related to vibration measurements. By definition, this uncertainty is mainly related to the measurement noise $\mathbf{N}$. Consequently, the likelihood function $p(\mathbf{X} \mid \mathbf{F})$ can be written under the following form:

$$
p(\mathbf{X} \mid \mathbf{F})=p(\mathbf{X}-\mathbf{H F} \mid \mathbf{N}),
$$

representing the probability of obtaining $\mathbf{X}-\mathbf{H F}=\mathbf{0}$ given the measurement noise $\mathbf{N}$.

If the noise is supposed to be due to multiple independent causes, then the likelihood function can be represented by a complex multivariate normal distribution with zero mean and precision $\tau_{n}$ :

$$
p\left(\mathbf{X} \mid \mathbf{F}, \tau_{n}\right)=\left[\frac{\tau_{n}}{\pi}\right]^{N} \exp \left[-\tau_{n}\|\mathbf{X}-\mathbf{H F}\|_{2}^{2}\right],
$$

where $N$ is the number of measurement points.

\subsubsection{Choice of the prior probability distribution}

The prior probability distribution reflects the uncertainty related to the unknown excitation field $\mathbf{F}$. Actually, it can be seen as a measure of the a priori knowledge of the experimenter on the sources to identify.

For practical reasons, the excitation field $\mathbf{F}$ is supposed to be a real random vector, whose components are independent and identically distributed random variables following a Generalized Gaussian distribution. As a result, the prior probability distribution is written:

$$
p\left(\mathbf{F} \mid \tau_{s}, q\right)=\left[\frac{q}{2 \Gamma(1 / q)}\right]^{M} \tau_{s}^{\frac{M}{q}} \exp \left[-\tau_{s}\|\mathbf{F}\|_{q}^{q}\right],
$$

where:

- $q$ is the shape parameter of the distribution. Its value is defined in the interval $] 0,+\infty[$;

- $\|\bullet\|_{q}$ is the $\ell_{\mathrm{q}}-$ norm or quasi-norm, if $q \geq 1$ and $q<1$ respectively;

- $\tau_{s}$ is the scale parameter of the distribution, which can be viewed as a generalized measure of the precision of the distribution;

- $M$ is the number of reconstruction points;

- $\Gamma(x)=\int_{0}^{+\infty} t^{x-1} e^{-t} d t$ is the gamma function. 
It should be noted that the choice of a multivariate generalized Gaussian distribution allows a great flexibility for describing prior knowledge of the sources to identify. Formally, sparse excitation fields are promoted for $q \leq 1$, while distributed excitation fields are favored for $q=2[\overline{6}]$. In practical situations, the possible values of the shape parameter are bounded in the interval $] 0,2]$.

\subsection{Summary and application}

From the explanations given above, the standard Bayesian formulation of the reconstruction problem finally writes:

$$
p\left(\mathbf{F} \mid \mathbf{X}, \tau_{n}, \tau_{s}, q\right) \propto p\left(\mathbf{X} \mid \mathbf{F}, \tau_{n}\right) p\left(\mathbf{F} \mid \tau_{s}, q\right) .
$$

This formulation is said standard, because it leads to Tikhonov-like regularizations, which correspond to the MAP estimate of Eq. (6). To explore the posterior probability distribution, $10^{5}$ samples are drawn from Eq. (6). Fig. 2 and Tables 1 and 2 present the excitation fields and the estimated values of the forces $F_{1}$ and $F_{2}$ obtained using either with a proper choice of $q(q=0.5)$ [see Fig. 2a] or with a poor choice of $q(q=2)$ [see Fig. 2b]. It should be noted that optimal values of $\tau_{n}$ and $\tau_{s}$ are strongly related to the value of $q$ and are computed accordingly as explained in section 3 . Here, $\left(\tau_{n}, \tau_{s}\right)=\left(2.77 \times 10^{15}, 1.48\right)$ for $q=0.5$ and $\left(\tau_{n}, \tau_{s}\right)=\left(2.87 \times 10^{15}, 12.44\right)$ for $q=2$.

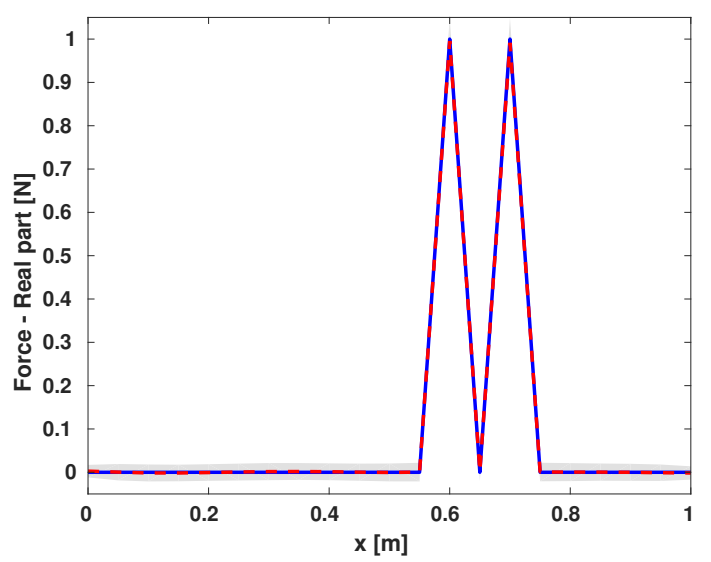

(a)

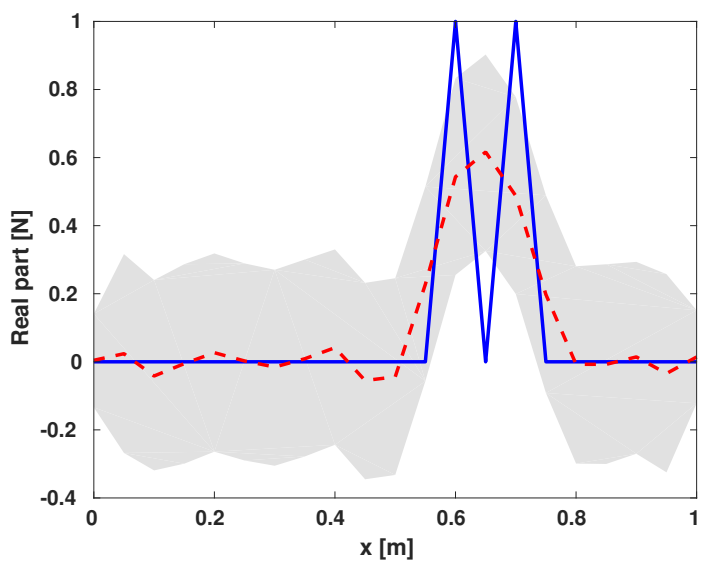

(b)

Figure 2: Standard Bayesian formulation - Real part of the reconstructed force vector (a) with a proper choice of $q$ $(q=0.5)$ and (b) a poor choice of $q(q=2)-(-)$ Reference, $(--)$ Median of the samples and ( $) 95 \%$ credible interval

\begin{tabular}{cccc}
\hline Parameter & Median & Mode & $95 \%$ CI \\
\hline$F_{1}$ & 0.996 & 0.996 & {$[0.942,1.049]$} \\
$F_{2}$ & 0.991 & 0.993 & {$[0.934,1.048]$} \\
\hline
\end{tabular}

Table 1: Standard Bayesian formulation - Summary of the inference result on the model parameters for $q=0.5$

Several interesting conclusions can be drawn at the light of the results presented in Fig. 2 and Tables 1 and 2. First of all, the choice of the supposedly known parameters have a significant 


\begin{tabular}{cccc}
\hline Parameter & Median & Mode & $95 \%$ CI \\
\hline$F_{1}$ & 0.543 & 0.543 & {$[0.255,0.833]$} \\
$F_{2}$ & 0.447 & 0.447 & {$[0.193,0.778]$} \\
\hline
\end{tabular}

Table 2: Standard Bayesian formulation - Summary of the inference result on the model parameters for $q=2$

impact on the quality of reconstruction. Indeed, a proper choice of the shape parameter leads to a very good agreement of the reference field with the median of the samples, associated with sharp posterior uncertainty region characterizes by the related $95 \%$ credible interval. On the contrary, a poor choice of the shape parameter leads to a disappointing result, since the shape of the median of the samples does not allow to discriminate the two point forces. Furthermore, the posterior uncertainty region is quite large, which is closely related to the value of the shape parameter $q$. All things being equal, the larger the value of the shape parameter is, the larger is the area of the posterior uncertainty region.

The standard formulation gives information on the credibility of the identified excitation field given the measured vibration field $\mathbf{X}$, the precisions $\tau_{n}$ and $\tau_{s}$ and the shape parameter $q$. Actually, this is the main drawback of the standard formulation, because the quality of the inference is conditioned to the knowledge of the precisions and the shape parameter. If their values are poorly chosen, then the resulting inference won't be representative of the actual distribution. As a consequence, it is compulsory to determine near-optimal values of $q, \tau_{n}$ and $\tau_{s}$ if one wants to perform a relevant statistical inference.

\subsection{Extended Bayesian formulation}

To alleviate the limitations of the standard formulation, the approach generally adopted in the literature consists in considering the precisions $\tau_{n}$ and $\tau_{s}$ as random variables, while letting fixed the shape parameters $q$. If we further consider the precisions as independent variables, the following extended formulation is obtained:

$$
p\left(\mathbf{F}, \tau_{n}, \tau_{s} \mid \mathbf{X}, q\right) \propto p\left(\mathbf{X} \mid \mathbf{F}, \tau_{n}\right) p\left(\mathbf{F} \mid \tau_{s}, q\right) p\left(\tau_{n}\right) p\left(\tau_{s}\right),
$$

where $p\left(\tau_{n}\right)$ and $p\left(\tau_{s}\right)$ are the prior probability distributions of the precisions $\tau_{n}$ and $\tau_{s}$ respectively.

\subsubsection{Choice of the prior probability distribution of the precisions}

The choice of the priori probability distributions $p\left(\tau_{n}\right)$ and $p\left(\tau_{s}\right)$ is first limited to distribution having a strictly positive support, because the precisions $\tau_{n}$ and $\tau_{s}$ are real positive numbers. The common choice, made in the literature, is the Gamma distribution. The reason for this is rather clear, since the conjugate prior for the precision of a generalized Gaussian distribution is a Gamma distribution [7]. Practically, the Gamma distibution is defined by:

$$
\mathcal{G}(\tau \mid \alpha, \beta)=\frac{\beta^{\alpha}}{\Gamma(\alpha)} \tau^{\alpha-1} \exp (-\beta \tau) \quad \text { with } \quad \alpha>0, \beta>0
$$

where $\alpha$ and $\beta$ are respectively the scale parameter and the rate parameter of the distribution. 
However, the use of a Gamma distribution is questionable, since it has been chosen for mathematical convenience and does not reflect any real prior information on the precisions, except their positiveness. That is why, the prior distribution on $\tau_{n}$ and $\tau_{s}$ should be as minimally informative as possible [8]. To this end, one sets $\alpha_{n}=\alpha_{s}=1$ and $\beta_{n}=\beta_{s} \rightarrow 0$.

\subsubsection{Summary and application}

Considering the previous choice, the extended Bayesian formulation of the reconstruction problem is given by:

$$
p\left(\mathbf{F}, \tau_{n}, \tau_{s} \mid \mathbf{X}, q\right) \propto p\left(\mathbf{X} \mid \mathbf{F}, \tau_{n}\right) p\left(\mathbf{F} \mid \tau_{s}, q\right) p\left(\tau_{n} \mid \alpha_{n}, \beta_{n}\right) p\left(\tau_{s} \mid \alpha_{s}, \beta_{s}\right),
$$

where $\left(\alpha_{n}, \beta_{n}\right)$ are the hyperparameters related to the precision $\tau_{n}$, while $\left(\alpha_{s}, \beta_{s}\right)$ are the hyperparameters related to the precision $\tau_{s}$.

This extended formulation has given rise, when $q=2$, to the augmented Tikhonov regularization [9]. This method provides a point estimate corresponding to a critical point of the opposite of the logarithm of the posterior probability distribution. The main advantage of this approach is to determine the regularized solution and the precision simultaneously using an iterative process.

As previously done, $10^{5}$ samples have been drawn from the MCMC algorithm presented in section 3 to explore the posterior probability distribution. Fig. 3 presents the excitation fields obtained using either a proper choice of $q(q=0.5)$ [see Fig. 3a] or with a poor choice of $q$ $(q=2)$ [see Fig. 3b], while Tables 3 and 4 summarize the inference results on each parameter of the model for $q=0.5$ and $q=2$ respectively.

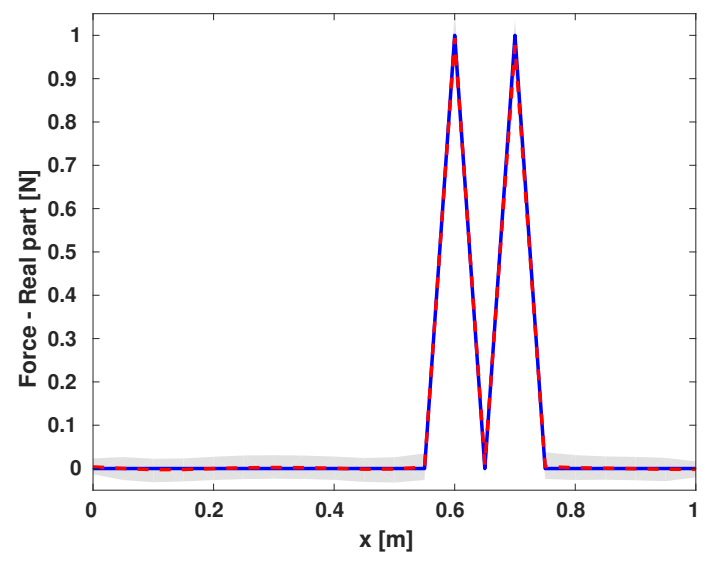

(a)

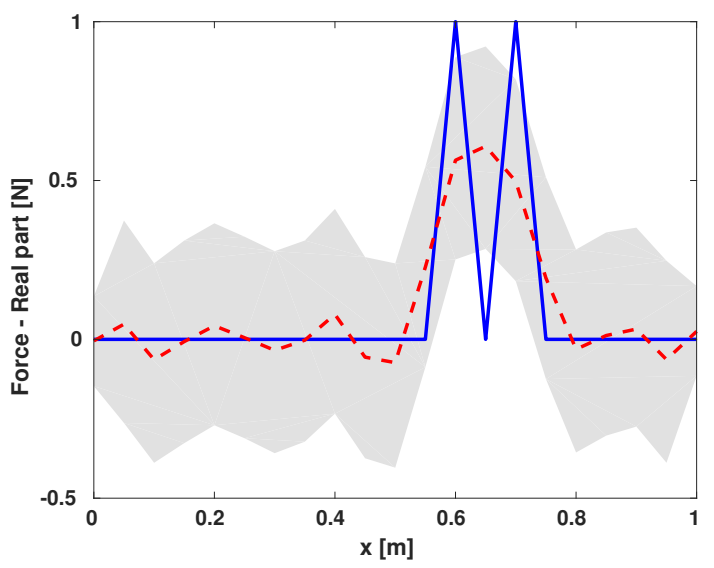

(b)

Figure 3: Extended Bayesian formulation - Real part of the reconstructed force vector (a) with a proper choice of $q(q=0.5)$ and (b) a poor choice of $q(q=2)-(-)$ Reference, $(--)$ Median of the samples and ( ) $95 \%$ credible interval

Obtained results clearly show that the quality of the inference strongly depends on a proper choice of the shape parameter $q$. Consequently, when setting $q=2$, as classically done in the literature to perform the inference, one takes the risk to draw erroneous conclusions if the structure is actually excited by localized sources, even if the precisions are estimated from Bayesian inference. 


\begin{tabular}{cccc}
\hline Parameter & Median & Mode & $95 \%$ CI \\
\hline$F_{1}$ & 0.998 & 0.998 & {$[0.946,1.053]$} \\
$F_{2}$ & 0.987 & 0.987 & {$[0.931,1.041]$} \\
$\tau_{n}$ & $4.87 \times 10^{15}$ & $4.67 \times 10^{15}$ & {$[2.87,7.67] \times 10^{15}$} \\
$\tau_{s}$ & 10.44 & 10.19 & {$[7.42,14.21]$} \\
\hline
\end{tabular}

Table 3: Extended Bayesian formulation - Summary of the inference result on the model parameters for $q=0.5$

\begin{tabular}{cccc}
\hline Parameter & Median & Mode & $95 \%$ CI \\
\hline$F_{1}$ & 0.565 & 0.565 & {$[0.253,0.889]$} \\
$F_{2}$ & 0.497 & 0.497 & {$[0.181,0.817]$} \\
$\tau_{n}$ & $4.29 \times 10^{15}$ & $4.04 \times 10^{15}$ & {$[2.17,7.80] \times 10^{15}$} \\
$\tau_{s}$ & 10.37 & 9.81 & {$[5.38,17.88]$} \\
\hline
\end{tabular}

Table 4: Extended Bayesian formulation - Summary of the inference result on the model parameters for $q=2$

\subsection{Complete Bayesian formulation}

The applications of standard and extended Bayesian formulations have pointed out the need for properly defining the value of the shape parameter $q$. However, choosing a priori relevant value is far from an easy task for non-experienced user. That is why, it is interesting to infer the shape parameter from a Bayesian analysis. Practically, this is done by considering this parameter as a random variable. In doing so, one obtains the complete Bayesian formulation:

$$
p\left(\mathbf{F}, \tau_{n}, \tau_{s}, q \mid \mathbf{X}\right) \propto p\left(\mathbf{X} \mid \mathbf{F}, \tau_{n}\right) p\left(\mathbf{F} \mid \tau_{s}, q\right) p\left(\tau_{n} \mid \alpha_{n}, \beta_{n}\right) p\left(\tau_{s} \mid \alpha_{s}, \beta_{s}\right) p(q),
$$

where $p(q)$ is the prior probability distribution of the shape parameter $q$.

\subsubsection{Choice of the prior probability distribution of the shape parameter}

The only available information is that the value of the shape parameter is bounded and positive. In absence of more precise knowledge on this parameter, the probability distribution is not only chosen to reflect the available information but also for its mathematical tractability. A probability distribution that meets these requirements is the truncated Gamma distribution defined by:

$$
\mathcal{G}_{T}\left(q \mid \alpha_{q}, \beta_{q}, l_{b}, u_{b}\right)=\frac{\Gamma\left(\alpha_{q}\right)}{\gamma\left(\alpha_{q}, \beta_{q} u_{b}\right)-\gamma\left(\alpha_{q}, \beta_{q} l_{b}\right)} \mathcal{G}\left(q \mid \alpha_{q}, \beta_{q}\right) \mathbb{I}_{\left[l_{b}, u_{b}\right]}(q),
$$

where:

- $\mathcal{G}\left(q \mid \alpha_{q}, \beta_{q}\right)$ is the Gamma distribution defined in Eq. (8);

- $\mathbb{I}_{\left[l_{b}, u_{b}\right]}(q)$ is the truncation function defined between the lower bound $l_{b}$ and the upper bound $u_{b}$. More precisely, this function simply writes:

$$
\mathbb{I}_{\left[l_{b}, u_{b}\right]}(q)= \begin{cases}1 & \text { if } q \in\left[l_{b}, u_{b}\right] \\ 0 & \text { otherwise }\end{cases}
$$


- $\gamma(s, x)=\int_{0}^{x} t^{s-1} \exp (-t) d t$ is the lower incomplete Gamma function.

Here, the choice of the truncated Gamma distribution has been made for mathematical convenience, because other continuous truncated distribution could have theoretically been used. To avoid biasing the inference, the shape of the prior distribution needs to be weakly informative. Here, this means that the hyperparameters should be defined such that $\alpha_{q}=1$ and $\beta_{q} \rightarrow 0$. On the other hand, even if the lower and upper bounds $l_{b}$ and $u_{b}$ can theoretically take any positive value, one knows that the value of $q$ practically lies in the interval ]0,2]. For this particular reason, we set $l_{b}=0.05$ and $u_{b}=2.05$.

\subsubsection{Summary and application}

From the above considerations, the complete Bayesian formulation is given by:

$$
p\left(\mathbf{F}, \tau_{n}, \tau_{s}, q \mid \mathbf{X}\right) \propto p\left(\mathbf{X} \mid \mathbf{F}, \tau_{n}\right) p\left(\mathbf{F} \mid \tau_{s}, q\right) p\left(\tau_{n} \mid \alpha_{n}, \beta_{n}\right) p\left(\tau_{s} \mid \alpha_{s}, \beta_{s}\right) p\left(q \mid \alpha_{q}, \beta_{q}, l_{b}, u_{b}\right) .
$$

To explore the posterior probability distribution, $10^{5}$ samples have been drawn from the MCMC algorithm presented in section 3 . Fig. 4 presents the excitation field obtained after performing the inference, while Table 5 summarizes the inference results on the model parameters.

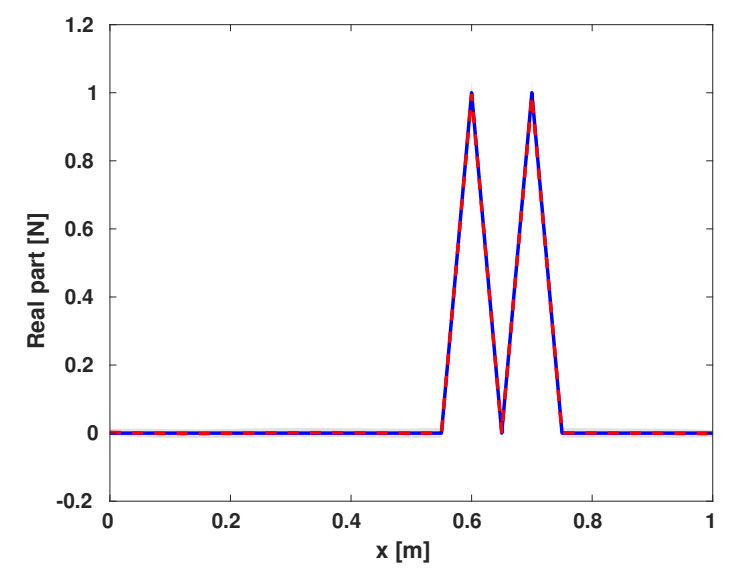

Figure 4: Complete Bayesian formulation - Real part of the reconstructed force vector $-(-)$ Reference, $(--)$ Median of the samples and $(\square) 95 \%$ credible interval

\begin{tabular}{cccc}
\hline Parameter & Median & Mode & $95 \%$ CI \\
\hline$F_{1}$ & 0.995 & 0.995 & {$[0.959,1.033]$} \\
$F_{2}$ & 0.992 & 0.992 & {$[0.952,1.030]$} \\
$\tau_{n}$ & $5.02 \times 10^{15}$ & $4.69 \times 10^{15}$ & {$[3.02,7.84] \times 10^{15}$} \\
$\tau_{s}$ & 11.51 & 11.28 & {$[8.62,14.80]$} \\
$q$ & 0.26 & 0.24 & {$[0.14,0.44]$} \\
\hline
\end{tabular}

Table 5: Complete Bayesian formulation - Summary of the inference result on the model parameters

As expected, obtained results clearly show that the inference performed from the complete Bayesian formulation is able to provide parameters estimates as well as a quantification of 
the posterior uncertainty on those parameters. In particular, it can be stressed that the median of the reconstructed excitation field agrees very well with the reference one. Furthermore, it could be added that the size of the associated $95 \%$ credible interval is small, meaning that the reconstructed excitation field is highly probable given the mechanical model and the measured vibration field.

\section{BAYESIAN INFERENCE - MCMC ALGORITHM}

In the previous section, the main features of each Bayesian formulation have been studied without describing the algorithm behind the results presented. Actually, a Gibbs sampler have been implemented to explore the posterior probability distribution of each Bayesian formulations. To render the presentation of the proposed sampler more concise, the proposed Gibbs sampler is derived for the complete Bayesian formulation, since the samplers associated to the standard and the extended Bayesian formulations are, in fact, only particular cases of this more general sampler.

The implementation of a Gibbs sampler requires the knowledge of the full conditional probability distributions. For the complete Bayesian formulation, one has for:

- the shape parameter $q$ :

$$
p\left(q \mid \mathbf{X}, \mathbf{F}, \tau_{n}, \tau_{s}\right) \propto \frac{\tau_{s}^{\frac{M}{q}}}{\Gamma(1 / q)^{M}} q^{\alpha_{q}+M-1} \exp \left[-\beta_{q} q-\tau_{s}\|\mathbf{F}\|_{q}^{q}\right] \mathbb{I}_{\left[l_{b}, u_{b}\right]}(q) ;
$$

- the precision $\tau_{s}$ :

$$
p\left(\tau_{s} \mid \mathbf{X}, \mathbf{F}, \tau_{n}, q\right) \propto \mathcal{G}\left(\tau_{s} \mid \alpha_{s}+\frac{M}{q}, \beta_{s}+\|\mathbf{F}\|_{q}^{q}\right)
$$

- the precision $\tau_{n}$ :

$$
p\left(\tau_{n} \mid \mathbf{X}, \mathbf{F}, \tau_{s}, q\right) \propto \mathcal{G}\left(\tau_{n} \mid \alpha_{n}+N, \beta_{n}+\|\mathbf{X}-\mathbf{H F}\|_{2}^{2}\right)
$$

- the force vector $\mathbf{F}$ :

$$
p\left(\mathbf{F} \mid \mathbf{X}, \tau_{n}, \tau_{s}, q\right) \propto \exp \left[-\tau_{n}\|\mathbf{X}-\mathbf{H F}\|_{2}^{2}-\tau_{s}\|\mathbf{F}\|_{q}^{q}\right],
$$

which corresponds to the standard Bayesian formulation.

From the previous full conditional probability distributions, we can derive the following Gibbs sampler:

1. Set $k=0$ and initialize $q^{(0)}, \tau_{s}^{(0)}, \tau_{n}^{(0)}$ and $\mathbf{F}^{(0)}$;

2. Draw $N_{s}$ samples from full conditional distributions

for $k=1: N_{s}$

a. $\operatorname{draw} q^{(k)} \sim p\left(q \mid \mathbf{X}, \mathbf{F}^{(k-1)}, \tau_{n}^{(k-1)}, \tau_{s}^{(k-1)}\right)$ 

b. $\operatorname{draw} \tau_{s}^{(k)} \sim p\left(\tau_{s} \mid \mathbf{X}, \mathbf{F}^{(k-1)}, \tau_{n}^{(k-1)}, q^{(k)}\right)$
c. draw $\tau_{n}^{(k)} \sim p\left(\tau_{n} \mid \mathbf{X}, \mathbf{F}^{(k-1)}, \tau_{s}^{(k)}, q^{(k)}\right)$
d. $\operatorname{draw} \mathbf{F}^{(k)} \sim p\left(\mathbf{F} \mid \mathbf{X}, \tau_{n}^{(k)}, \tau_{s}^{(k)}, q^{(k)}\right)$

\section{end for}

3. Monitor the convergence of the Markov chains

In the next of this section, each step of the Gibbs sampler is detailed.

\subsection{Initialization of the sampler}

In the present contribution, the sampler is initialized from a starting point having a reasonably high probability. Here, the initial force vector $\mathbf{F}^{(0)}$ is obtained from the MAP estimate of the standard Bayesian formulation, which is defined by [see Ref. [3] for more details]:

$$
\mathbf{F}^{(0)}=\underset{\mathbf{F}}{\operatorname{argmin}}\|\mathbf{X}-\mathbf{H F}\|_{2}^{2}+\lambda^{(0)}\|\mathbf{F}\|_{q^{(0)}}^{q^{(0)}}
$$

where $\lambda^{(0)}=\tau_{s}^{(0)} / \tau_{n}^{(0)}$ is the regularization parameter.

To obtain a relevant initial force vector, it is necessary to determine reasonable values of the shape parameters $q^{(0)}$ and the precisions $\tau_{s}^{(0)}$ and $\tau_{n}^{(0)}$. Practically, the values of the shape parameter $q^{(0)}$ can be chosen without any calculation using the indications given in section 2.2.2. However, because $q^{(0)}$ can take any value in the range ]0,2], the solution of the previous optimization problem has generally no closed-form expression and hence the optimal value of the regularization parameter $\lambda^{(0)}$ can not be directly computed from automatic selection procedures such as the L-curve principle [10].

Practically, the optimization problem is solved using an Iteratively Reweighted Least Squares (IRLS) algorithm [11]. From a Bayesian standpoint, the IRLS procedure can be viewed as a transformation of the Generalized Gaussian prior into a multivariate Gaussian-like prior. More precisely, one has:

$$
\begin{aligned}
p\left(\mathbf{F} \mid \mathbf{X}, \tau_{s}^{(0)}, q^{(0)}\right) & =p\left(\mathbf{F} \mid \tau_{s}^{(0)}, q^{(0)}\right) \\
& \propto p\left(\mathbf{F} \mid \mathbf{W}, \tau_{s}^{(0)}\right) \\
& \propto \exp \left[-\tau_{s}^{(0)}\left\|\mathbf{W}^{1 / 2} \mathbf{F}\right\|_{2}^{2}\right],
\end{aligned}
$$

where $\mathbf{W}$ is a diagonal global weighting matrix depending explicitly on $\mathbf{F}$ and $q^{(0)}$. This explains why Eq. (18) can only be solved using an iterative procedure.

At each iteration $\mathrm{j}$ of the IRLS algorithm, one has to solve:

$$
\mathbf{F}^{(0, j)}=\underset{\mathbf{F}}{\operatorname{argmin}}\|\mathbf{X}-\mathbf{H F}\|_{2}^{2}+\lambda^{(0, j)}\left\|\mathbf{W}^{(j)^{1 / 2}} \mathbf{F}\right\|_{2}^{2},
$$

until the convergence is reached. 
After convergence of the iterative process, the optimal force vector $\mathbf{F}^{(0)}$, the global weighting matrix $\mathbf{W}$, as well as the optimal value of the regularization parameter $\lambda^{(0)}$, that has been updated at each iteration using automatic selection procedure such as the L-curve principle, are obtained. Consequently, to complete the initialization step it remains to determine the values of $\tau_{n}^{(0)}$ and $\tau_{s}^{(0)}$. Here, we follow the approach proposed by Pereira et al. [12] consisting in finding the optimal values of $\tau_{n}^{(0)}$ and $\tau_{s}^{(0)}$ given the measured vibration field $\mathbf{X}$ only using a Bayesian approach. With the proposed parametrization, one has:

$$
\tau_{s}^{(0)}=\frac{N}{\mathbf{X}^{H}\left(\lambda^{(0)} \mathbf{I}+\mathbf{H W}^{-1} \mathbf{H}^{H}\right)^{-1} \mathbf{X}} \quad \text { and } \quad \tau_{n}^{(0)}=\frac{\tau_{s}^{(0)}}{\lambda^{(0)}} .
$$

\subsection{Drawing samples for the shape parameter}

The Gibbs sampler is generally the first choice for conditionally conjugate models, where samples can be drawn directly from each conditional probability distribution. Unfortunately, this is not the case for the shape parameters $q$, for which the conditional distribution is not standard. To bypass this difficulty, we propose to update the value of the shape parameter using a Hamiltonian Monte Carlo (HMC) step, because it limit the random walk behavior of the sampler.

In HMC, the Hamiltonian function can be written as follows:

$$
H(q, s)=U(q)+K(s)
$$

where $U(q)$ is called the potential energy and is defined as:

$$
U(q)=-\log \left[p\left(q \mid \mathbf{X}, \mathbf{F}^{(k-1)}, \tau_{n}^{(k-1)}, \tau_{s}^{(k-1)}\right)\right],
$$

while $K(s)$ is called the kinetic energy and is chosen for mathematical convenience. Usually, it is defined such that:

$$
K(s)=-\log [\mathcal{N}(s \mid 0,1)]=\frac{1}{2} s^{T} s+\text { const } .
$$

In this framework, $q$ and $s$ define the state space of the dynamical system and can be viewed as the position and the momentum respectively. The evolution of the state $(q, s)$ over the time $t$ is governed by the following system of equations:

$$
\left\{\begin{array}{l}
\frac{d q}{d t}=\frac{\partial H(q, s)}{\partial s} \\
\frac{d s}{d t}=-\frac{\partial H(q, s)}{\partial q}
\end{array}\right.
$$

Practically, Eq. (25) is solved using the leapfrog method. Starting from an initial state $\left(q_{0}, s_{0}\right)$, it allows determining a proposal state $\left(q^{\star}, s^{\star}\right)$, while taking into account the constraints on the parameter $q$. Once a proposal state is obtained, one has to decide to accept it as a new state with probability:

$$
P=\min \left(1, \exp \left[H\left(q_{0}, s_{0}\right)-H\left(q^{\star}, s^{\star}\right)\right]\right),
$$

or to reject it with probability $1-P$. 


\subsection{Drawing samples for the precisions}

The conditional probability distribution for the precisions $\tau_{n}$ and $\tau_{s}$ are actually gamma distributions. Consequently, samples can be easily drawn from standard statistical packages.

\subsection{Drawing samples for the force vector}

At first sight, drawing samples from $p\left(\mathbf{F} \mid \mathbf{X}, \tau_{s}^{(k)}, \tau_{s}^{(k)}, q^{(k)}\right)$ seems a difficult task. However, the IRLS algorithm allows writing when the iterative process has converged:

$$
\begin{aligned}
p\left(\mathbf{F} \mid \mathbf{X}, \tau_{n}^{(k)}, \tau_{s}^{(k)}, q^{(k)}\right) & \propto \exp \left[-\tau_{n}^{(k)}\|\mathbf{X}-\mathbf{H F}\|_{2}^{2}-\tau_{s}^{(k)}\left\|\mathbf{W}^{1 / 2} \mathbf{F}\right\|_{2}^{2}\right] \\
& \propto \mathcal{N}_{c}\left(\mathbf{F} \mid \boldsymbol{\mu}_{\mathbf{F}}, \boldsymbol{\Sigma}_{\mathbf{F}}\right),
\end{aligned}
$$

where $\mathcal{N}_{c}(\mathbf{z} \mid \boldsymbol{\mu}, \boldsymbol{\Sigma})$ is the (circurlarly-symmetric) complex multivariate Gaussian distribution with mean $\boldsymbol{\mu}$ and covariance matrix $\boldsymbol{\Sigma}$. Here, the mean $\boldsymbol{\mu}_{\mathrm{F}}$ and the covariance matrix $\boldsymbol{\Sigma}_{\mathrm{F}}$ are expressed as:

$$
\boldsymbol{\mu}_{\mathbf{F}}=\tau_{n}^{(k)} \boldsymbol{\Sigma}_{\mathbf{F}} \mathbf{H}^{H} \mathbf{X} \quad \text { and } \quad \boldsymbol{\Sigma}_{\mathbf{F}}=\left(\tau_{n}^{(k)} \mathbf{H}^{H} \mathbf{H}+\tau_{s}^{(k)} \mathbf{W}\right)^{-1}
$$

Once $\boldsymbol{\mu}_{\mathbf{F}}$ and the covariance matrix $\boldsymbol{\Sigma}_{\mathbf{F}}$ are obtained, samples of the force vector $\mathbf{F}^{(k)}$ can be easily drawn. A widely used method for drawing samples from a complex multivariate Gaussian distribution consists in:

1. generating a complex random vector $\mathbf{v} \sim \mathcal{N}_{c}(\mathbf{v} \mid \mathbf{0}, \mathbf{I})$ from two real normal random vectors $\mathbf{v}_{\mathbf{1}} \sim \mathcal{N}\left(\mathbf{v}_{\mathbf{1}} \mid \mathbf{0}, \mathbf{I}\right)$ and $\mathbf{v}_{\mathbf{2}} \sim \mathcal{N}\left(\mathbf{v}_{\mathbf{2}} \mid \mathbf{0}, \mathbf{I}\right):$

$$
\mathbf{v}=\frac{\mathbf{v}_{\mathbf{1}}+j \mathbf{v}_{\mathbf{2}}}{\sqrt{2}}
$$

where $j=\sqrt{-1}$ is the imaginary unit;

2. determining any matrix $\mathbf{L}$ satisfying the relation $\mathbf{L} \mathbf{L}^{H}=\boldsymbol{\Sigma}_{\mathbf{F}}$. This can be typically done using either a Cholesky decomposition or a spectral decomposition;

3. computing the new sample $\mathbf{F}^{(k)}=\boldsymbol{\mu}_{\mathbf{F}}+\mathbf{L} \mathbf{v}$.

\subsection{Convergence diagnostics}

In the present contribution, all the inferences have been performed from one single chain for each monitored parameter. More precisely, we have monitored the convergence of the precisions $\left(\tau_{n}, \tau_{s}\right)$ and the shape parameter $q$. The procedure used for convergence monitoring is based on the combination of two complementary diagnostics:

1. the Raftery-Lewis diagnostic [13] that estimates the total run length and the burn-in period;

2. the Geweke diagnostic [14] that tests the null hypothesis that the Markov chain is in the equilibrium distribution and produces z-statistics for each estimated parameter.

Practically, the Raftery-Lewis is first applied to the chains to monitor. Then, the Geweke diagnostic is applied to the resulting chains. 


\subsection{Samplers for the standard and the extended Bayesian formulations}

The MCMC algorithm for the complete Bayesian formulation can be used to derive the MCMC algorithms corresponding to the standard and the extended Bayesian formulations. Indeed, the MCMC algorithm related to the extended Bayesian formulation is obtained by not considering the step $2 \mathrm{a}$ of the Gibbs sampler (i.e. by fixing the values of $q$ ), while the sampler associated to the standard Bayesian formulation is derived from the general Gibbs sampler by computing steps 1 and $2 \mathrm{c}$ only (i.e. by considering fixed values for $\tau_{n}, \tau_{s}$ and $q$ ).

\section{CONCLUSIONS}

In structural dynamics, regularization approaches can be used to deal with source identification problems. Although widely used and deeply studied, these methods generally provide only point estimates. In other words, there is no information on the uncertainty about the regularized solution given the measured data and the mechanical model. However, such information is of primary interest for industrial applications, in which it is essential to guarantee the quality of obtained results. In this contribution, three Bayesian formulations of the force reconstruction problem have been proposed to tackle this issue and an original hybrid Gibbs sampler has been implemented to perform the inferences. One of the merits of proposed sampler is to be flexible enough to deal with each formulation and provide estimates and uncertainty quantification of all the parameters of the formulation considered. The proposed numerical example clearly highlight the benefits and the limitations of the proposed formulations. In particular, it has been shown that the standard and extended formulations should be used when precise information about the problem is available. On the contrary, the complete formulation should be used in case of too vague information about the problem. In this case, however, the solution is obtained at a higher computational cost.

\section{REFERENCES}

[1] A.N. Tikhonov, Regularization of incorrectly posed problems. Soviet Mathematics, 4, 1624-1627, 1963.

[2] A.N. Thite, D. J. Thompson, The quantification of structure-borne transmission paths by inverse methods. Part 2 : Use of regularization techniques. Journal of Sound and Vibration, 264 (2), 433-451, 2003.

[3] M. Aucejo, O. De Smet, Bayesian source identification using local priors. Mechanical Systems and Signal Processing, 66-67, 120-136, 2016.

[4] S. Geman, D. Geman, Stochastic Relaxation, Gibbs Distributions, and the Bayesian Restoration of Images. IEEE Transactions on Pattern Analysis and Machine Intelligence, PAMI-6 (6), 721-741, 1984.

[5] S. Duane, A. D. Kennedy, B. J. Pendleton, D. Roweth, Hybrid Monte Carlo. Physics Letters B, 195 (2), 216-222, 1987.

[6] S. Boyd, L. Vandenberghe, Convex optimization. Cambridge University Press, 2004.

[7] C. M. Bishop, Pattern recognition and machine learning. Springer, 2006.

[8] P. D. Hoff, A first course in Bayesian statistical methods. Springer-Verlag New York, 2009. 
[9] B. Bin, J. Zhou, Augmented Tikhonov regularization. Inverse Problems, 25 (2), 025001 25 pages, 2008.

[10] P. C. Hansen, Rank-Deficient and Discrete Ill-Posed Problems: Numerical Aspects of Linear Inversion. SIAM, 1998.

[11] P. Rodriguez, B. Wohlberg, An Iteratively Weighted Norm Algorithm for Total Variation Regularization. 2006 Asilomar Conference on Signals, Systems, and Computers, Pacific Grove, USA, 2006.

[12] A. Pereira, J. Antoni, Q. Leclere, Empirical Bayesian regularization of the inverse acoustic problem. Applied Acoustics, 97, 11-29, 2015.

[13] A. E. Raftery, S. M. Lewis, One long run with diagnostics: Implementation strategies for Markov chain Monte Carlo. Statistical Science, 7 (4), 493-497, 1992.

[14] J. Geweke, Evaluating the Accuracy of Sampling-Based Approaches to Calculating Posterior Moments. Bayesian Statistics 4: Proceedings of the Fourth Valencia International Meeting, Valencia, Spain, 1992. 